\title{
Prevalencia de procesos y patologías atendidos por los servicios de emergencia médica extrahospitalaria en España
}

\author{
Prevalence of processes and pathologies dealt with by the pre- \\ hospital emergency medical services in Spain
}

\author{
A. Pacheco', S. Burusco ${ }^{2}$, M.V. Senosiáin ${ }^{2}$
}

\section{RESUMEN}

Este estudio se ha realizado contactando con 26 Servicios de Emergencia Médica (SEMEx), vía encuesta tabulada, obteniendo datos finales de 22. La población de cobertura atendida: 42.538 .730 habitantes. El número total de llamadas: 7.656 .768 , con 711.228 intervenciones. El número de dispositivos operativos al día: unidad de vigilancia intensiva (UVI-móviles): 329 , vehículos de intervención rápida (VIR) 20, ambulancias sanitarias 39 y helicópteros medicalizados 39 (5 de ellos 24 horas: Islas Canarias y Baleares, y Castilla-LaMancha) y 3 aviones. Intervenciones: UVI-móvil: 94,3\%, helicópteros medicalizados: $1,4 \%$ y ambulancias sanitarias: $4,3 \%$. El número de médicos de emergencias/día: 388, personal enfermería 427. Las patologías atendidas, agrupadas según clasificación CIE-9-MC: grupo V-psiquiatría: 3,7\%, grupo VI-neurológico: 2,7\%, grupo VII-cardiovascular: $9,3 \%$, grupo VIII-respiratorio: $3,2 \%$, grupo XVII-lesiones envenenamientos: $23,1 \%$, grupo IX-digestivo: $1,3 \%$, grupos X-XI-genito-urinario y gineco-obstetricia: 1,4\%, grupo XVI-otros mal definidos: 40,1\%. Los datos de los grupos específicos estudiados (de los SEMEx que los aportaron) fueron: dolor torácico-disnea: $3,7 \%$, ictus/accidente cerebrovascular agudo: $0,9 \%$, alteración conciencia: $7,7 \%$, síncope-lipotimia: $2,5 \%$, autolesiones: $2.654(0,4 \%)$. Total grupos patológicos y sindrómicos: $59,9 \%$ y total de otros mal definidos: $40,1 \%$. De las patologías consideradas de mayor relevancia en urgencias-emergencias: síndrome coronario agudo: $3,1 \%$, arritmias: $3,2 \%$, paro cardiaco: $1,7 \%$, paro respiratorio: $0,16 \%$, traumatismos totales: $34,9 \%$, politraumatismos: $1,16 \%$, traumatismo cráneo encefálico: $1,77 \%$, intoxicaciones: $5,1 \%$, agresiones: $6,9 \%$, quemados: $0,26 \%$.

Palabras clave. Servicios de Emergencia Médica. Medicina de urgencias. Prevalencia. España.

\begin{abstract}
This study was carried out by contacting $26 \mathrm{Me}-$ dical Emergency Services (MES), through survey, with final data obtained from 22. Population covered: $42,538,730$. Total number of calls: $7,656,768$, with 711,228 interventions. Number of operational forces: intensive care units (mobile-ICUs): 329, rapid intervention vehicles (RIV) 20 , health ambulances 39 and medical helicopters 39 ( 5 of them 24 hour: Canary and Balearic Islands and Castille-La Mancha) and 3 airplanes. Interventions: mobile-ICUs: $94.3 \%$, medical helicopters: $1.4 \%$ and health ambulances: $4.3 \%$. Number of A\&E doctors/day: 388, nursing personnel 427 . Pathologies dealt with, grouped according to ICE-9-MC: V-psychiatry group: 3.7\%, VI-neurological group: 2.7\%, VII-cardiovascular group: 9.3\%, VIII-respiratory group: $3.2 \%$, XVII-lesions poisonings group: $23.1 \%$, IX-digestive group: $1.3 \%, \mathrm{X}$-XI-genital-urinary and gynaecologyobstetrics groups: $1.4 \%$, XVI-group of other ill-defined pathologies: $40.1 \%$. Specifically studied groups (from the MESs that provided them) were: thoracic paindyspnea: $3.7 \%$, ictus/acute cerebrovascular accident: $0.9 \%$, alteration in consciousness: $7.7 \%$, syncope-blackout: $2.5 \%$, self-inflicted lesions: $2,654(0.4 \%)$. Total of pathological and syndrome groups: $59.9 \%$ and total of other ill-defined pathologies: $40.1 \%$. Of the pathologies considered to be of greater relevance in A\&E: acute coronary syndrome: $3.1 \%$, arrhythmias: $3.2 \%$, cardiac arrest: $1.7 \%$, respiratory stoppage: $0.16 \%$, total traumatisms: $34.9 \%$, traumatisms: $1.16 \%$, cranioencephalic traumatism: $1.77 \%$, intoxications: $5.1 \%$, aggressions: $6.9 \%$, burns: $0.26 \%$.
\end{abstract}

Key words. Emergency medical services. Emergency medicine. Prevalence. Spain.
1. Gerencia de Urgencias, Emergencias y Transporte Sanitario GUETS-Sescam. Servicio de Salud de Castilla La Mancha. Consorcio público SCIS-Emergencia Ciudad Real.

2. Servicio de Urgencias Extrahospitalarias-SUE. Servicio Navarro de Salud-Osansunbidea. Pamplona.

\section{Correspondencia}

Andrés Pacheco Rodríguez

Jaraíz, 1

13300 Valdepeñas. Ciudad Real

E-mail: apacheco1701@telefonica.net 


\section{INTRODUCCIÓN}

Los servicios de emergencia médica extrahospitalaria (SEMEx) son también denominados servicios de emergencia médica (SEM), servicios de urgencia médica (SUM) o servicios integrales de urgencia (SIU). Su denominación primigenia se estableció en Estados Unidos de Norteamérica en la década de los años $70^{1-3}$ donde ya se establecieron los componentes y estructura de los mismos. Tuvo su correspondencia en Euro$\mathrm{pa}$, inicialmente en el área franco-germana, también en esa misma década ${ }^{4-6}$

A diferencia con los SEMEx norteamericanos (Emergency medical services, EMS), que operan con ténicos en emergencias (TEM) o paramédicos, los europeos del área citada incorporan profesionales de la medicina (Service d'Aide Medicale Urgente, SAMU y Service d'Incendie et Secours, $S D I S)$. En España, tras algunas experiencias pioneras aisladas y localizadas a nivel municipal y de otro ámbito geográfico, en los servicios de fuego y rescate ${ }^{7-9}$, empiezan a estructurarse, con recursos móviles y capacidad de transporte medicalizado en los diferentes servicios de salud, a principios de los años $90^{10,11}$, con un ámbito de operatividad geodemográfica más amplio (estructuras provinciales, áreas sanitarias, e incluso regionales). En estos casos, la mayoría surgieron como una reorganización de los servicios especiales de urgencias, de atención primaria y otros.

Con las transferencias de las competencias de la sanidad estatal a las comunidades autónomas, regiones o países, estos SEMEx llegan a adquirir cobertura regional. En algunas áreas coexisten con SEMEx de servicios de fuego y rescate, protección civil o servicios municipales configuradas, en general, como gerencias especiales de urgencias y emergencias y en otras áreas como empresas, fundaciones o consorcios públicos, dependientes de las consejerías de sanidad, de los servicios de salud, e incluso de servicios de fuego y rescate. La mayoría de SEMEx cuentan con sus propios centros de coordinación de urgencias y emergencias (CECUEs). Los teléfonos de acceso, en los SEMEx dependientes de los servicios de salud, fueron inicialmente o son el 061. Los dependientes de otras administraciones fueron o son: 006-1006, 080, 085, 088, 092, etc. Las estructuras de coordinación en torno a los CECUEs regionales o provinciales, que disponen del teléfono 1-12 genérico para todo tipo de emergencias, dependen de departamentos de interior, presidencia, administraciones públicas y otros. En unos territorios se ha integrado físicamente el personal propio de los CECUEs de los SEMEx y en otros no. Por otro lado, diversos servicios regionales de salud disponen de dispositivos móviles con capacidad de traslado medicalizado a las patologías de urgencia y emergencia, basados en su estructura de atención primaria (ejemplo, en Andalucía, los dispositivos de cuidados críticos y urgencias, asimilados a servicios de urgencias de atención primaria, se cuenta con amplio dispositivo de intervención y transporte asistido, basado en 128 ambulancias medicalizadas). Y, por lo tanto, la asistencia a las emergencias, en algunos territorios, está fundamentada en ambas estructuras y en otras.

A nivel internacional, diversos autores, sociedades científicas, grupos de trabajo y otras instituciones han publicado documentos relativos a la necesidad de implantación de los SEMEx, recomendaciones de configuración y estructura, experiencias pioneras y datos de su estructura y actividad ${ }^{1-3 .}$

En España, con una experiencia desde la creación de los primeros SEMEx que ronda ya las dos décadas, se han publicado también documentos de proyectos, de sistemas, modelos y estructuras de los servicios de asistencia médica urgente (SAMU) o sistemas integrales de urgencia (SIU) o servicios de emergencia médica (SEM) ${ }^{12-19}$, también documentos de procesos y actividad de estos diferentes SEMEx, tanto terrestres $^{11,20,21}$ como aéreos $^{22}$ así como documentos de consenso de expertos ${ }^{23}$. Incluso se han publicado datos de actividad y prevalencia de patologías de áreas geográficas concretas en las que actúan los SEMEx, analizando retrospecivamente los últimos 20 años desde su implantación (área de Tomelloso, Ciudad Real ${ }^{24}$. Los datos de es- 
tructura, dispositivos asistenciales y actividad de diferentes SEMEx se han publicado en diversos formatos y presentaciones

En este estudio se analiza exclusivamente la patología primaria, atendida in situ por los diferentes SEMEx del estado español, dada la dificultad para obtener los datos globales de los diferentes dispositivos asistenciales integrados en los servicios de urgencia de atención primaria (SUAPs, PACs, DCCUs, etc) que atienden, en su quehacer diario, también a las urgencias y emergencias sanitarias. Consideramos complejo el comparar los diferentes SEMEx en España, como así lo han estimado ya otros autores a nivel internacional ${ }^{25}$.

Y por ello este estudio pretende mostrar los datos de actividad y la prevalencia de las patologías atendidas por los SEMEx, pero no compararlos. Existen publicaciones donde se establecen medidas de rendimiento de los SEMEx y estándares de acreditación para los mismos, tanto a nivel internacional como en España ${ }^{26,27}$.

El objetivo principal del presente estudio es conocer la prevalencia de procesos y patologías que atienden de forma primaria (in situ) los SEMEx en España y así objetivar datos de las citadas patologías o procesos sindrómicos en base a las intervenciones reales. Como objetivos secundarios se incluyen otros datos de actividad y estructura, como el número de llamadas solicitando asistencia médica urgente, la cobertura poblacional, la dimensión de sus recursos y tipo de sus dispositivos asistenciales, tanto terrestres como aéreos.

\section{MATERIAL Y MÉTODOS}

Se han revisado los trabajos previos publicados sobre indicadores de estructura y actividad de los diferentes SEMEx en España, así como las memorias oficiales de los mismos, accesibles en la web y en otros tipos de documentos. El motor de búsquedas bibliográficas de la revista Emergencias, revista científica de la Sociedad Española de Medicina de Urgencias y Emergencias (SEMES) ha sido también de gran utilidad ${ }^{28}$.
También se han buscado referencias bibliográficas en las bases Medline-PubMed, Web Of Knowlegde (WOK-ISI-Thompson), Excerpta Medica-Embase, Indice Médico Español, Base de Datos Teseo de Tesis Doctorales, LILACS, Scielo.

Las palabras claves para la estrategia de búsqueda en la web fueron: Emergency Medical Services, EMS, Service d'Aide Medicale Urgente, SAMU, Service d'Incendie et Secours, SDIS, Helicopter Emergency Medical Services, urgencias, emergencias, servicios de emergencia médica, servicios y sistemas integrales de urgencia, servicios especiales de urgencia, ambulancias, UVI-móviles, ambulancias medicalizadas, helicópteros medicalizados, transporte aéreo sanitario, helicópteros sanitarios, servicios de salud, servicio de urgencia médica, servicio de ayuda médica urgente, atención integral urgencias, emergencias médicas, asistencia médica urgente, transporte medicalizado, urgencias médicas extrahospitalarias, aerotransporte sanitario, transporte aéreo medicalizado, HEMS, extrahospitalario, prehospitalario, unidades de soporte vital avanzado, USVA, ambulancias de soporte vital avanzado, ASVA, SEM, SEMEx, SIU, SAMU, protección civil, fuego y rescate, bomberos, prevalencia, patologías, actividad, memoria, informe.

Tras un primer análisis de los datos obtenidos de las citadas memorias anuales, se objetivó la dificultad de agrupar los mismos para obtener una idea homogénea de los procesos más prevalentes atendidos por los diferentes SEMEx, agrupados en ítems homologables o asimilados. Por ello se elaboraron expresamente dos tablas más un cuestionario adicional. La denominación de las tablas fue la siguiente: tabla 1 , acceso, cobertura poblacional, recursos, dotación e indicadores de actividad; y la tabla 2 prevalencia de procesos y patologías en la atención urgente extrahospitalaria. En esta última se desglosa, del total de patologías atendidas, las que lo hicieron con recursos terrestres y con recursos aéreos.

Las variables utilizadas para su cumplimentación están fundamentadas en la Clasificación Internacional de Enfermedades 
CIE-9-Modificación Clínica (CIE-9-MC), pero adaptada a grupos generales y diagnósticos concretos de especial interés en la atención médica a la urgencia/emergencia extrahospitalaria ${ }^{29}$.

Se notifica expresamente que, entre las adaptaciones tabulares a la CIE-9-MC que hemos realizado, la patología enfermedad cerebro-vascular aguda (ACVA) (VII-7) fue incluida en el grupo VI (enfermedades del sistema nervioso y órganos de los sentidos): en nuestra tabla, grupo neurología. Si bien hemos obtenido los datos concretos referidos al ACVA. También se han agrupado los grupos X (enfermedades del aparato genitourinario) y el XI (complicaciones del embarazo, parto y puerperio), ya que fueron desglosadas en nuestras tablas en dos grupos: grupo urología y grupo gineco-obstetricia. Se han obtenido datos específicos adicionales como E-19: suicidio y lesiones autoinflingidas (grupo E, clasificación suplementaria de causas externas de lesiones y envenenamientos). Y los sindrómicos específicamente tabulados como dolor torácico-disnea, alteración de concienciacoma-mareo-convulsiones, síncope-lipotimia-pérdida de conocimiento y, por último, el grupo otros (no definidos), que corresponderían al grupo XVI (síntomas, signos y estados mal definidos) de la CIE-9-MC.

Las patologías específicas, incluidas en sus grupos correspondientes, que fueron expresamente tabuladas son: (síndrome coronario agudo/infarto agudo de miocardio (SCA/lam), arritmias, paro cardiorrespiratorio, politraumatismo, traumatismo cráneo encefálico (TCE), intoxicaciones, agresiones, autolesiones (suicidio-parasuicidio), quemados, paro respiratorio, e ictus/ACVA. Estos grupos diagnósticos y diagnósticos concretos pueden objetivarse en las figuras 1 y 2 .

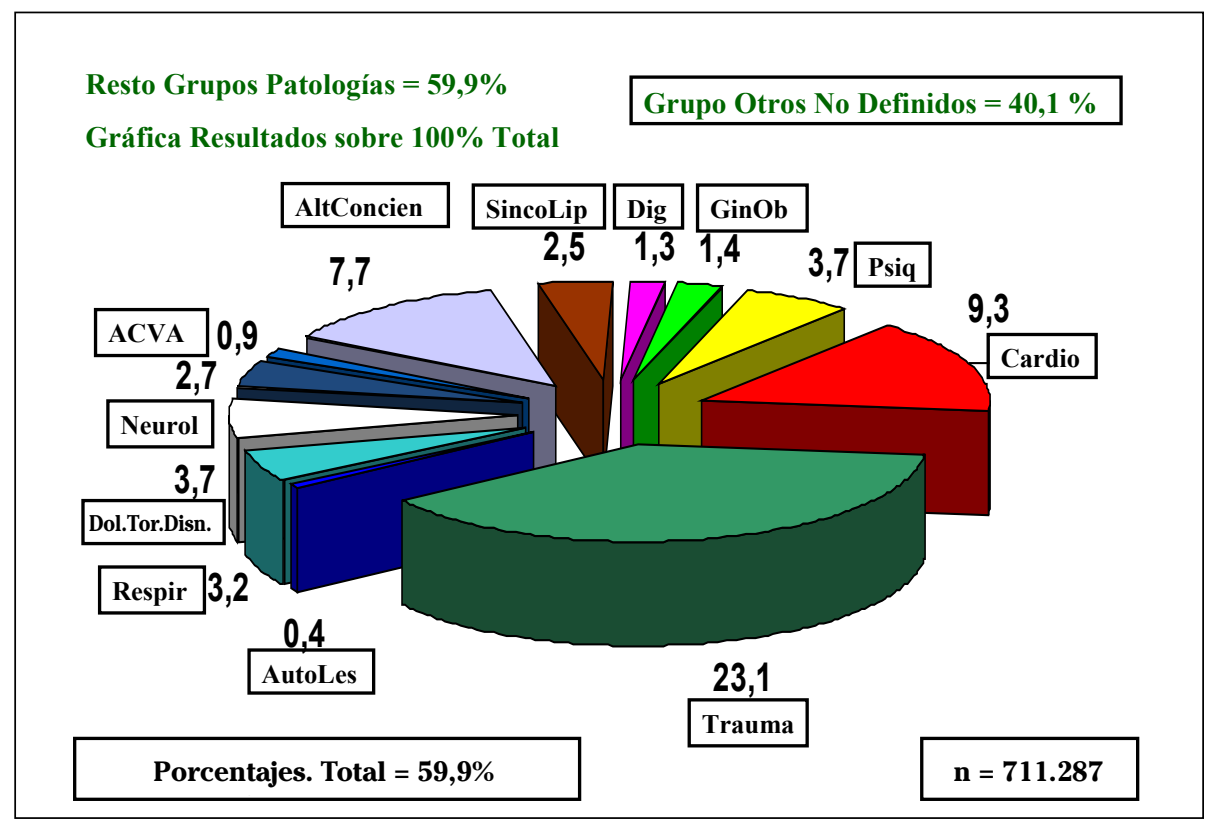

Grupos basados en la clasificación CIE-9-MC: Psq: grupo V (Psiquiatría). Neurol: grupo VI (Neurológico). Cardio: grupo VII (Cardiovascular). Respir: grupo VIII (Respiratorio). Trauma: grupo XVII (Lesiones y envenenamientos: Dig: grupo IX (Digestivo). GinOb: grupos X y XI conjuntos (genito urinario y ginecobstetricia)

Grupos específicos segregados de la CIE-9-MC fueron: Dol.Tor.Disn.: dolor torácico-disnea. ACVA: ictus/accidente vasculo cerebral. AltConcienc: alteración de la conciencia (y coma-mareo-convulsiones). SincoLIp: síncope-lipotimia-pérdida de conocimiento. AutoLes: autolesiones (suicidio y lesiones autoinflingidas).

Figura 1. Prevalencia de patologías atendidas por Servicios de Emergencia Médica Extrahospitalaria (SEMEx) en España. 
Patologías Específicas atendidas por SEMEx

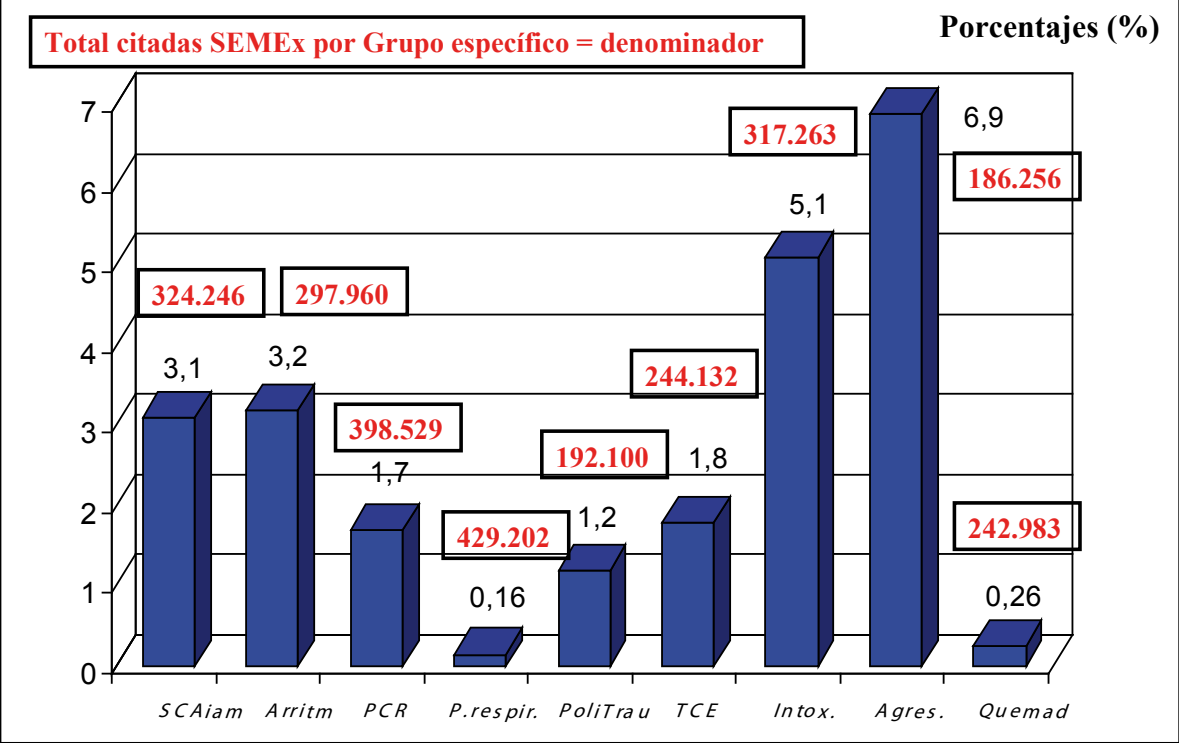

SCAiam: síndrome coronario agudo/infarto agudo de miocardio. Arritm: arritmias. PCR: paro cardio respiratorio. Prespir: paro respiratorio. PoliTrau: politraumatismo. TCE: traumatismo cráneo encefálico. Intox: intoxicaciones y envenenamientos. Agres: agresiones. Quemad: quemaduras.

Figura 2. Prevalencia de patologías atendidas por Servicios de Emergencia Médica Extrahospitalaria (SEMEx) en España. Patologías específicas.

La encuesta fue enviada por correo electrónico y en algún caso por correo postal. El seguimiento de su cumplimentación se realizó a través de vía telefónica y correo electrónico. Sólo un servicio respondió por correo postal, dado que la información que enviaban lo era en formato impreso y material audiovisual (CD$\mathrm{ROM})^{30}$. Los datos del resto de servicios de emergencia (cuatro) fueron obtenidos a través de accesos web $^{31-34}$.

\section{Limitaciones del estudio}

Cabe la posibilidad de no haber contactado con algún SEMEx de reciente creación, del que no tenemos conocimiento, sobre todo basados en estructuras municipales, de servicios de fuego y rescate, protección civil u otros. Tampoco se han estudiado los diferentes servicios de emergencia médica del ámbito privado. La minoría de SEMEx contactados que no cumplimentaron el cuestionario tabulado, también se incluyen en el estudio, basándonos en los datos que hemos obtenido de sus memorias oficiales y datos de la publicación de Pesqueira y $\mathrm{col}^{21}$ y contactos no oficiales con personal operativo de estos servicios.

\section{RESULTADOS}

De los veintiséis SEMEx consultados, diecinueve han cumplimentado la tabla de recursos y actividad y dieciocho las tablas de prevalencia de patologías de los cuestionarios enviados por correo electrónico. Otros enviaron memorias o datos, en formato no tabulado como les fue requerido, pero que han sido considerados válidos, al poder obtener datos objetivo del estudio. Del resto de SEMEx, los datos fueron obtenidos a través de los accesos web ya citados. Los datos presentados en este 
estudio son referidos a 2008, salvo alguna excepción.

Por lo tanto los datos absolutos de los grupos de patologías CIE-9-MC y grupos sindrómicos, no representan los datos reales de todos los SEMEx de España, porque no todos aportaron el dato concreto en todas las celdas. Por ello, estos datos absolutos y sus porcentajes serán referidos al total de datos aportados por los distintos SEMEx para cada ítem en su celda correspondiente. O sea, el denominador es la suma total de dígitos aportados para ese ítem. De los datos presentados, el total de las intervenciones sí corresponde al total de las reales informadas.

En la tabla 1 (recursos y actividad), en los datos que figuran en la tabulación como intervención UVI-móvil, se incluyen los datos aportados de intervención de VIR (vehículo de intervención rápida), ya que sólo lo han aportado como desglosados el SUMMA-112 de Madrid y SEM-061 de Catalunya.

Tabla 1. Servicios de emergencia médica extrahospitalaria España (SEMEx). Cobertura. Dispositivos, personal asistencial y actividad.

\begin{tabular}{|c|c|c|c|c|c|c|c|c|c|c|c|}
\hline $\begin{array}{c}\text { SEMEx } \\
\text { Cobertura } \\
\text { Población }\end{array}$ & $\begin{array}{c}\text { Tfnos. } \\
\text { Acceso } \\
\text { Número }\end{array}$ & $\begin{array}{c}\text { Llamadas** } \\
\text { Urgencias } \\
\text { Emergencias }\end{array}$ & $\begin{array}{c}\text { UVI-m } \\
\text { Totales } \\
\text { día }\end{array}$ & $\begin{array}{c}\text { VIR } \\
\text { Totales } \\
\text { día }\end{array}$ & $\begin{array}{c}\text { AmbuSan } \\
\text { Totales } \\
\text { día }\end{array}$ & $\begin{array}{c}\text { HEMS } \\
+ \text { avión } \\
\#\end{array}$ & $\begin{array}{c}\text { Médicos } \\
\text { día }\end{array}$ & $\begin{array}{l}\text { DUEs } \\
\text { día }\end{array}$ & $\begin{array}{c}\text { Intervención } \\
\text { UVI-m + } \\
\text { VIR }\end{array}$ & $\begin{array}{c}\text { Intervención } \\
\text { DUEs } \\
\text { AmbuSan }\end{array}$ & $\begin{array}{c}\text { Intervención } \\
\text { HEMS } \\
\&\end{array}$ \\
\hline $\begin{array}{l}42.538 .730^{*} \\
\text { habitantes }\end{array}$ & $\begin{array}{l}061 \\
080 \\
092 \\
112\end{array}$ & $7.656 .768^{* *}$ & 329 & 20 & 39 & $\begin{array}{c}39 \#+ \\
3\end{array}$ & 388 & 427 & 671.004 & 30.507 & 9.776 \\
\hline
\end{tabular}

*Andalucía dispone de un dispositivo de ambulancias medicalizadas (128), complementario a EPES, que da cobertura al resto población.

**Llamadas totales que no incluyen los SEMEx Málaga bomberos-080, SAMU-Asturias-061, Ceuta-INGESA-061 ni SAMERLas Rozas-092

\# HEMS-helicópteros medicalizados: 39 (5 de ellos 24 horas: Islas Canarias y Baleares, y Castilla-La Mancha) y 3 aviones medicalizados (Baleares y Canarias)

\& Incluye traslados asistidos primarios y secundarios (interhospitalarios).

VIR: Vehículo medicalizado de intervención rápida (también denominado VEMS-vehículo emergencia medicalizado) AmbuSan: ambulancias sanitarias con DUE-enfermería a bordo (también denominados ECAs-equipos coordinación avanzada- y USVA-I -unidades de soporte vital intermedio-

En la tabla 1 los datos referidos a las ambulancias sanitarias o unidades de soporte vital avanzado intermedio (AmbuSan), no medicalizada y dotadas de personal de enfermería, aparecen entre paréntesis (n) y están diferenciados de los registros de UVI-móvil y del helicóptero medicalizado (HEMS). Sin embargo, sí se han incluido sus datos de actividad en los gráficos de prevalencia de patologías atendidas, ya que no han sido desglosados por los diferentes SEMEx. Los SEMEx que aportaron los datos sobre número de dispositivos y actividad de las AmbuSan fueron EPES-061Andalucía, Emergentziak-Oxakidetza (Servicio Vasco de Salud) y Servicio de Urgencias Canario (SUC-061-Gestión Sanitaria de Canarias) y SEM-061 Catalunya.
Los datos globales de la población de cobertura atendida por todos los SEMEx es de 42.538.730 habitantes. El número total de llamadas de demanda sanitaria urgente fue de 7.656.768, con un total de intervenciones de 711.228. El número total de UVI-móviles operativas es de 329, VIRes 20, ambulancias sanitarias 39 y helicópteros medicalizados 39 (de los cuales 5 operan las 24 horas -Islas Canarias y Baleares, y Castilla-La Mancha-). El total de intervenciones de UVI-móvil fue de 671.004, helicópteros medicalizados 9.776 y ambulancias sanitarias 30.507. El número de profesionales por día de la medicina, asistenciales operativos de UVI-móvil fue de 388 y el de personal de enfermería fue de 427. 
En relación con las patologías atendidas, agrupadas según clasificación CIE9-MC, los datos fueron para el total de 711.287 intervenciones: grupo V (psiquiatría) 26.406 (3,7\%), grupo VI (neurológico) $19.236(2,7 \%)$, grupo VII (cardiovascular) 66.279 (9,3\%), grupo VIII (respiratorio) $22.706(3,2 \%)$, grupo XVII (lesiones y envenenamientos) $164.270(23,1 \%)$, grupo IX (digestivo) $9.562(1,3 \%)$, grupos X y XI conjuntos (genito urinario y gineco-obstetricia) $10.166(1,4 \%)$, grupo XVI (otros mal definidos) $284.844(40,1 \%)$.

Los datos de los grupos específicos segregados de la CIE-9-MC fueron: dolor torácico-disnea: 26.240 (3,7\%), ictus/ACVA $6.210(0,9 \%)$ alteración de la conciencia (y coma-mareo-convulsiones) $54.676(7,7 \%)$, síncope-lipotimia-pérdida de conocimiento $18.042(2,5 \%)$, autolesiones $2.654(0,4 \%)$ (Fig. 1). Total grupos patológicos y sindrómicos: $59,9 \%$ y total de otros mal definidos: $40,1 \%$.

De las patologías consideradas de mayor relevancia en el ámbito de la medicina de urgencia y emergencia extrahospitalaria se han analizado las siguientes (ya incluidas en sus respectivos grupos CIE-9-MC o sindrómicos tabulados en este estudio). Con la observación de que el denominador de la fórmula para obtener el porcentaje es la suma de los datos referidos a ese ítem concreto, es el de la suma del total de intervenciones (ya conocido) de los SEMEx que aportan datos sobre estos grupos patológicos:
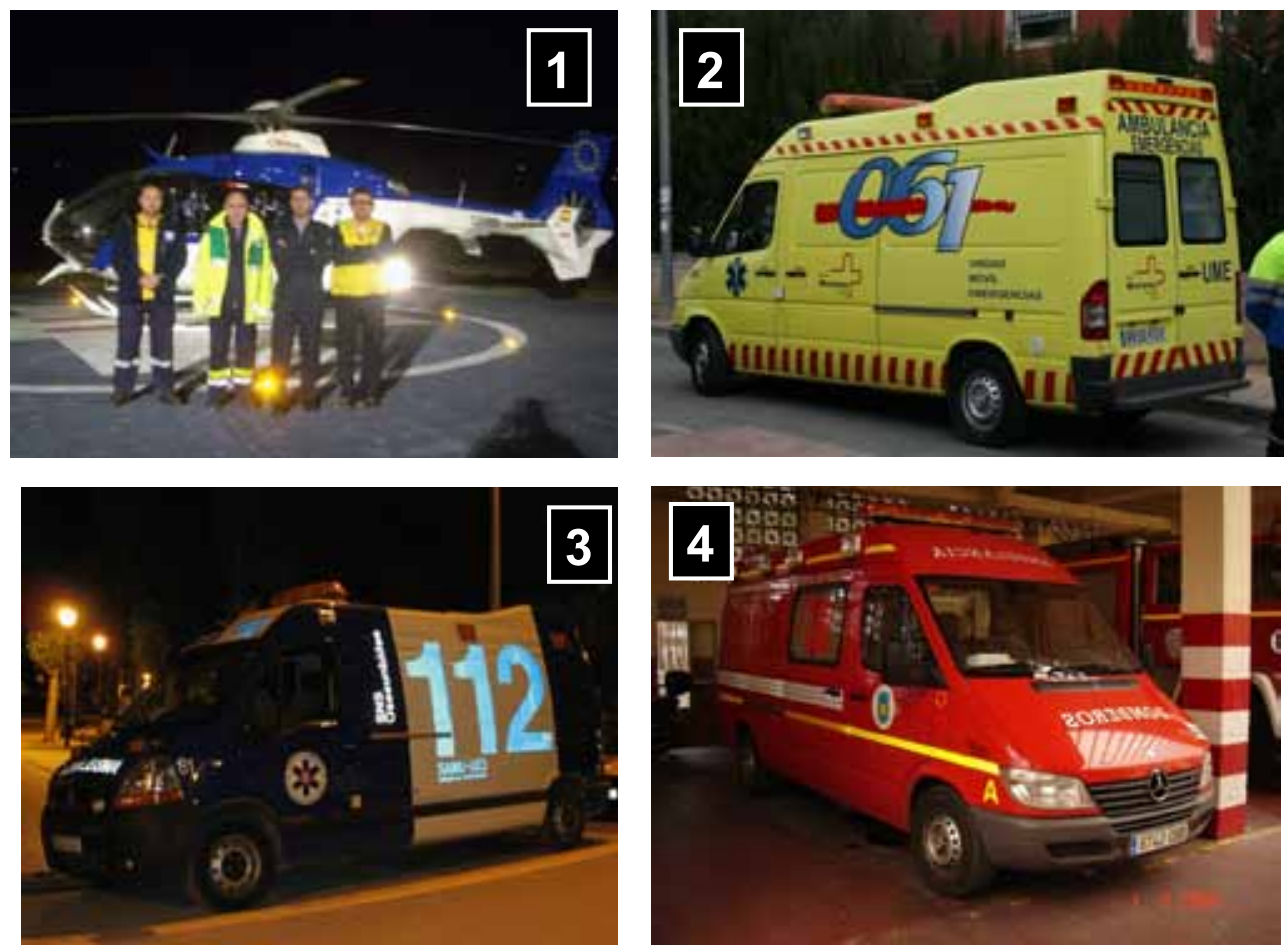

1. Helitransporte medicalizado, servicio nocturno. Gerencia de Urgencias, Emergencias y Transporte Sanitario, GUETSSescam, Servicio de Salud de Castilla La Mancha.

2. UME Emergencias-061, Servicio Murciano de Salud.

3. SAMU-UCI Osasunbidea-112, Servicio Navarro de Salud.

4. Ambulancia medicalizada, Bomberos-080, Ayuntamiento de Málaga.

Figura 3. Dispositivos asistenciales. Servicios de Emergencia Médica Extrahospitalaria (SEMEx) de España. 
Síndrome coronario agudo/infarto agudo de miocardio (SCA-IAM): 10.115/324.246 (3,1\%), arritmias: $9.645 / 297.960(3,2 \%)$, paro cardiaco: $6.598 / 398.529(1,7 \%)$, paro respiratorio: $702 / 429.202(0,16 \%)$, traumatismos totales: $169.360 / 484.187(34,9 \%)$, politraumatismos: $2.240 / 192.100(1,16 \%)$, traumatismo cráneoencefálico (TCE): 4.311/244.132 (1,77\%), intoxicaciones: $16.293 / 317.263$ (5,1\%), agresiones: 12.870/186.256 (6,9\%), quemados: $643 / 242.983 \quad(0,26 \%)$. Total $23,35 \%$ (excluyendo traumatismos totales, ya que incluyen al TCE y politrauma (Fig. 2). En la figura 3 se muestras imágenes de diversos SEMEx españoles.

\section{DISCUSIÓN}

Como se ha comentado en el apartado resultados, del total de los totales por grupos CIE-9-MC y grupos sindrómicos adaptados, un número indeterminado de estas patologías se encontrarían incluidas en el grupo XVI (otros mal definidos), lo cual induce a pensar que para incluir patologías clasificadas en la CIE-9-MC, más nuestra adaptación, han tenido muchas dificultades para obtener los datos concretos que les solicitamos dadas las diferentes plataformas tecnológicas y sistemas de codificación y explotación estadística. Por ello, casi la mitad de los datos de prevalencia de patologías atendidas por los SEMEx españoles están etiquetados, en este estudio como "otros mal definidos", lo cual resta capacidad para conocer más específicamente qué patologías concretas han sido atendidas. De los grupos CIE-9-MC (de los que fueron extraídos los ítems autolesiones y ACVA), y añadidos a la tabulación los grupos específicos dolor torácico-disnea, alteración de la conciencia, sincope-lipotimia, el denominador utilizado ha sido el total de intervenciones de los SEMEx. Pero no todos ellos aportaron el dato en todas sus celdas, por lo que los que no lo hicieron, esos datos probablemente estén incluídos en el grupo "otros mal definidos", que consideramos muy sobredimensionado.

Sin embargo, los resultados de los grupos específicos de patologías concretas, analizadas explícitamente (e incluidas en sus grupos correspondientes CIE-9-MC), son más fiables, porque representan datos de la realidad aportada por los diferentes SEMEx. Y sin que esto suponga que son los reales del Estado Español, porque el denominador de los mismos está basado en los datos que aportaron en esa celda concreta los SEMEx españoles, resulta sugerente su análisis del total de sus intervenciones. Así en el grupo SCA-IAM son datos que representan el 45\% (324.246) del total de las intervenciones totales: 711.287, arritmia: $42 \%$, paro cardiorrespiratorio: $56 \%$, trauma total: $68 \%$, (politrauma: $27 \%$, TCE: $34 \%$, incluidos en trauma total), intoxicaciones: $44 \%$, agresiones: $26 \%$, quemados: $34 \%$, paro respiratorio: $60 \%$ (Fig. 2).

Curiosamente, en un estudio referido a un área muy concreta (Tomelloso-Ciudad Real) en el que se analizaba la evolución de las patologías atendidas por el SEMEx Emergencia Ciudad Real y Gerencia de Urgencias, Emergencias y Trasporte Sanitario, GUETS-Sescam, del Servicio de Salud de Castilla La Mancha, durante 20 años se objetivaba un incremento de las intervenciones relacionadas con accidentes, intoxicaciones y violencia y una disminución en el síndrome coronario agudo. Este último ítem probablemente está relacionado con la apertura de un nuevo hospital en la citada localidad ${ }^{24}$.

No se han obtenido datos de prevalencia de patologías de dos SEMEx (aproximadamente 1.400 .00 habitantes) y tampoco pudieron cumplimentarlo otros dos SEMEx, por demora en contactar con sus directivos y plazos limitantes del proceso editorial (población estimada de 2.200.00 habitantes).

En resumen, en este estudio se han pretendido conocer los datos reales de prevalencia de patologías atendidas por los SEMEx españoles en todo el territorio geográfico. Sin embargo, por la dificultad en tabular los datos y los sistemas heterogéneos de codificación y explotación estadística, se puede concluir que este objetivo global ha sido alcanzado parcialmente. Sin embargo, en el estudio de patologías con- 
cretas consideradas de relevancia por su gravedad específica y prevalencia en las estadísticas sanitarias, se ha conseguido aproximar la realidad. Y esta realidad supone que el primer motivo de intervenciones de los SEMEx españoles es la patología traumática total por sí misma, seguida de agresión, intoxicaciones, y junto con quemaduras, suponen $47,2 \%$. En segundo lugar y seguida de lejos estarían las emergencias cardiacas (SCA-IAM, arritmias y paro cardiorrespiratorio) con el $8 \%$. Y como patologías específicas dentro del grupo de trauma total, el TCE: $1,77 \%$, el politrauma: $1,16 \%$. Por último, de las estudiadas específicamente el paro respiratorio: 0,16\%. Los grupos CIE-9-MC y grupos asociados en este estudio más prevalentes fueron: traumatismos y lesiones, cardiología, alteración de conciencia, dolor torácico-disnea, psiquiatría, respiratorio, neurológico, síncope-lipotimia, genito-urinario-obstetricia, digestivo, ACVA y autolesiones. A destacar que el $40,1 \%$ fueron clasificados como "otros mal definidos". Consideramos que este estudio, en los términos referidos en el texto, supone la presentación de unos datos de referencia para realizar las estimaciones que se consideren oportunas, de tipo logístico, asistencial, formativo, de investigación y otros.

\section{BIBLIOGRAFÍA}

1. Rockwood ChaA, Coleen MH, Farrington JD, Hampton DP, MotLey RE. History of emergency medical services in the United States. J Trauma 1976; 16: 299-308.

2. BoYD DR. The conceptual development of EMS systems in the United States. Part II. Emerg Med Serv 1982; 11: 26-35.

3. NARAD RA. Emergency Medical Services System Design. In: Roush WR, Fontanarosa PB. EMS/Prehospital Care. Emerg Med Clin North Am 1990; 8: 1-15.

4. Conseil de l’Europe. Étude comparative sur l'organization et le fonctionnement des Services d'Aide Médicale Urgente. Programme de recherche Médicales Coordonnées. 1986. Strasbourg 1990: 14.

5. BARRIER G. Histoire de l’Aide Médicale d’Urgence. Revue Annuelle de SAMU de Paris. SOGI communication. Paris 1992: 23-30.
6. STOLPE ED. Rentabilidad del transporte sanitario. En: Ruiz Boada FJ, Salinas Sánchez JC (coord.). I Jornadas cívico-militares de Sanidad. Madrid: Ministerio de Sanidad y Consumo; 1986: 241-244.

7. Ortega Carnicer J, Diarte JL, Ruiz de Alegría JR. Plan integral de urgencias en la provincia de Ciudad Real. Medicina Intensiva 1987; abril (extra): 107.

8. Maruenda A, Chuliá V, Belda F, Barberá M, RuFino A, Company R et al. Proyecto para la implantación de un Servicio de Ayuda Médica Urgente (SAMU) en la ciudad de Valencia. Rev Esp Anestesiol 1983; 30: 97-102.

9. Cester Martínez A. Bomberos en España. Situación de los servicios sanitarios. Protección Civil 1992; 14: 39-42.

10. Bermejo Pareja R, Álvarez Fernández JA, Curieses Asensio A, Fernández Onieva JM, García Pondal J, Margalef De Blas A. Hacia un sistema integral de urgencias en la Comunidad Autónoma de Madrid. Emergencias 1992; 4: 189-195.

11. Pacheco Rodríguez A, Álvarez García A, Hermoso Gadeo FE, Serrano Moraza A. Servicios de Emergencia Médica Extrahospitalaria en España (II). Emergencias 1998; 10: 245-254.

12. Hormaechea Cazón E, Perales Rodríguez de VIGURI N. Proyecto para un Plan de Atención Sanitaria de Urgencia (P.A.S.U.). FUNDESCO-Sociedad Española de Medicina Intensiva y Unidades Coronarias, SEMIUC. Madrid 1983.

13. Áluarez leiva C, Hormaechea Cazon E, Hernando Lorenzo A, Olavarria Govantes L, Puppo Moreno A. Sistemas de asistencia médica urgente (SAMU). Jano 1985; 651: 19-21.

14. Chulí CAmpos V. Asistencia Extrahospitalaria. Rev Esp Anestesiol 1985; 32 (Suppl. 1): 544548.

15. Alted E. Sistemas integrales de urgencia. Emergencias 1988; 1: 9-16.

16. GonZÁLEZ S. Sistemas integrados de Urgencia. Emergencias 1989; 1: 34-36.

17. Állvarez Fernández JA. Modelos de asistencia a las emergencias médicas en el medio extrahospitalario. Medifam 1995; 2: 80-86.

18. Pacheco Rodríguez A, Álvarez García A, Hermoso Gadeo FE, Serrano Moraza A. Servicios de Emergencia Médica Extrahospitalaria en España (I). Historia y fundamentos preliminares. Emergencias 1998; 10: 173-187.

19. García-Castrillo Riesgo L, Del Busto de Prado F. Modelo de atención integral a las urgencias. (editorial). Emergencias 2001; 13: 153-154. 
20. Actividad asistencial urgente en los Servicios de Emergencias. En: Sociedad Española de Medicina de Urgencias y Emergencias Urgencias Sanitarias en España: Situación actual y propuestas de mejora. Granada. Escuela Andaluza de Salud Pública 2003: 71-72.

21. Pesqueira Alonso EE, Juliani IzQuierdo P. Unidades de soporte vital avanzado en España 2008. Mapa de situación. Emergencias 2009; 21: 269-275.

22. Burillo Putze G, Herranz Duarte I, Álvarez FerNÁNDEZ JA. Helicopter emergency medical service in Spain. Air Med J 2001: 21-23.

23. Sociedad Española de Medicina de Emergencias. Grupo de Trabajo Interterritorial de Urgencias Médicas Extrahospitalarias. Asistencia extrahospitalaria a las urgencias médicas: documento de consenso. Emergencias 1994; 6: 2-7.

24. Pacheco Rodríguez A, Estudillo Mustafá A, Martín Fernández MC, De Buen Torralba J, Moreno Moreno MT, Iriarte Osa JM. En los últimos veinte años ¿ha variado la patología atendida por un servicio de emergencias? Emergencias 2008; 20: 290-291.

25. BOSSAERT LL. The complexity of comparing different EMS systems. A survey of EMS systems in Europe. Ann Emerg Med 1993; 22: 99-102.

26. Moore L. Performance Measurement in EMS. In: Swor RA, Pirrallo RG, editors. Improving quality in EMS. National Association of EMS Physicians. $2^{\text {nd }}$ edition. Dubuque, Iowa: Kendall Hunt Publishing Company 2005: 121-148.

27. Cartelle Pérez T, López Gomáriz A, Comes Sanroma M, Rodríguez Lorenzo M, Tejedor Fernández M, López-AndúJAR Aguiriano L. Estándares de acreditación para Servicios de Emergencias. Sociedad Española de Medicina de Urgencias y Emergencias. Madrid: Edicomplet-Grupo Saned; 2006.

28. Sociedad Española de Medicina de Urgencias y Emergencias, SEMES. Revista Emergencias. Disponible en: http://www.semes.org/revista_EMERGENCIAS.htm (varias consultas, de 01-07-2009 a 15-10-2009).

29. International classification of diseases (ICD) clinical modification. National Center for health Statistis (NCHS). World Health Organization WHO. Disponible en: http://www.cdc. gov/nchs/data/icd9/icdguide98.pdf (consultado 02-10-2009)

30. Anónimo. SUMMA-112. Madrid. Memoria SUMMA-112 y transporte sanitario. 20042007. Salud Madrid. Comunidad de Madrid. (memoria en CD-ROM). Madrid 2008. Depósito Legal: M-39990-2008.
31. Junta de Castilla y León. Portal de Salud de Castilla y León. Memorias de actividad. Emergencias Sanitarias de Castilla y León. Sacyl. Memoria de actividad 2007. Tríptico_GES_2007_Anverso [1]. pdf. Disponible en: http://www.salud.jcyl.es/sanidad/ $\mathrm{cm} /$ institucion/tkContent?pgseed $=12593$ 29269019\&idContent $=710484$ \&locale $=$ es ES\&textOnly=false (consultado 07-09-2009)

32. Rioja Salud. Memoria Área de Salud de la Rioja. 2007-2008. p. 28-29. Disponible en: http:/ www.riojasalud.es/ficheros/memorias/ srs2008.pdf (consultado 08-09-2009)

33. Gobierno de Cantabria. Consejería de Sanidad y Servicios Sociales. Servicio Cántabro de Salud. Gerencia de Atención Primaria 061 Cantabria. Actividad 2003. Pdf. Depósito Legal SA-680. 2004. Disponible en: http://www. scsalud.es/061/datos_actividad/descargar. php?Id3=1\&Id4=1 (consultado 08-09-2009)

34. Servicio de Salud del Principado de Asturias. SAMU Asturias. ¿Quiénes somos? Recursos materiales y organigramas. Disponible en: http://www.samuasturias.org/ (consultado 09-09-2009)

\section{Colaboradores}

Andalucía: Hilario Ruiz Rosales, Luis Olavarría Govantes, Miguel Angel Benítez, César Pinto Cosgaya

Aragón: Mabel Cano del Pozo, Armando Céster Martínez, José Antonio Cortés Ramas

Castilla La Mancha: Salvador Espinosa Ramírez, Francisco José Cano Muñoz.

Catalunya: Miquel Vidal i Domínguez, Xabier Escalada Roig, Muntsa Qunitillá Berge

Ceuta: Manuela Gómez

País Vasco: Txema Unanue Munduate

Extremadura: Susana Batres Gómez, Isabel Carpallo Darachona,

Galicia: Rosario García Rúa

Islas Baleares: Carlos Fuentes Nieto

Islas Canarias: Carmelo Duarte Merelo

Madrid: Ervigio Corral Torres, Dolores Murúa Petrobelly, Pedro Huertas Alcázar, María Rosario Carrillo Rodríguez

Melilla: Domingo Ortega López, Ana María Gómez Ane, Miguel Galera Martínez

Murcia: Diego Gómez Sánchez

Valencia: Rosa María Pérez Mencía, Purificación Sánchez Cuesta, Jesús Pitarch García

INAER: Jose Manuel Gutiérrez Rubio, Juan Antonio Sinisterra Aquilino 